\title{
The relaxation rate in hot-electron dynamics: beyond the first-order Born approximation in kinetic theory
}

\author{
J I Juaristi ${ }^{1}$, M Alducin ${ }^{2}$ and I Nagy ${ }^{2,3}$ \\ ${ }^{1}$ Departamento de Física de Materiales and Centro Mixto CSIC-UPV/EHU, Universidad del País \\ Vasco, Apartado 1072, 20080 San Sebastián, Spain \\ 2 Donostia International Physics Centre (DIPC), P Manuel de Lardizabal 4, 20018 San Sebastián, \\ Spain \\ ${ }^{3}$ Department of Theoretical Physics, Institute of Physics, Technical University of Budapest, \\ H-1521 Budapest, Hungary
}

Received 16 June 2003

Published 7 November 2003

Online at stacks.iop.org/JPhysCM/15/7859

\begin{abstract}
The validity of a perturbative approach to studying the lifetime of excited electrons in an electron gas is analysed in a range of metallic densities. The relaxation rate is calculated using a kinetic theory framework with secondorder Born and partial-wave approximations for the scattering amplitude. The comparison of the terms obtained by physically motivated Thomas-Fermitype potentials shows that the next-to-leading correction to the first-order result is small only for high densities of the electron gas. At low densities, a nonperturbative estimation of the relaxation rate is needed. An analytic expression of practical use which incorporates both the role of screening and scattering is derived. A comparison with experimental data obtained for an $\operatorname{Ag}(111)$ surface is made. The agreement found supports an inherent consistency, under the experimental conditions and down to $\left(E-E_{\mathrm{F}}\right)=0.4 \mathrm{eV}$, of the theoretical description based on a three-dimensional Fermi-liquid model.
\end{abstract}

\section{Introduction}

In the many-body problem of extended fermionic systems, like metals, an important quantity is the lifetime of an excited state. It is proved [1] to all orders of field-theoretical perturbation theory in a well-behaved interaction, that the inverse lifetime, i.e., the scattering rate, becomes $\left(1 / \tau_{\mathrm{ee}}\right)=a\left(E-E_{\mathrm{F}}\right)^{2}$ (atomic units are used throughout) for excited-state energy $E$ above, but close to, the Fermi level $E_{\mathrm{F}}$. The explanation behind the $\left(E-E_{\mathrm{F}}\right)^{2}$-character is simple: the Pauli principle restricts the available phase space for transitions. On the other hand, the determination of the $a$-prefactor requires further, reasonable approximations. The statistical and dynamical correlation effects are encoded in it.

Depending on the starting concept to model the interacting electron system of a metal, two main approximations are used. The first one $[2,3]$ considers the system as a medium 
described by its complex dielectric function, i.e., via the random-phase approximation (RPA). The other approximation, the kinetic model [3, 4], considers directly the screened particleparticle interaction. The physical mechanism for the decay of an excited state is the same in both treatments: the scattering rate is mediated by an effective interaction. In both approximations the energy and momentum conservations are satisfied.

Comparing the RPA-based dielectric and the scattering-amplitude-based kinetic frameworks, it was explicitly stated [3] that the kinetic one represents the more general attempt at $\varepsilon=\left(E-E_{\mathrm{F}}\right)<E_{\mathrm{F}}$ excess energy for a homogeneous system of fermions. The constraints under which the two approximations give the same expression for the lifetime were stressed recently: one has to implement the kinetic theory with the RPA-based instantaneous effective interaction and, in addition, apply a first-order Born scattering treatment [5]. Under these conditions the lowest-order result, denoted now by $a^{(1)}\left(\lambda, r_{\mathrm{s}}\right)$, is

$$
a^{(1)}\left(\lambda, r_{\mathrm{s}}\right)=\frac{1}{\pi v_{\mathrm{F}}} \frac{1}{\lambda^{3}}\left(\arctan \gamma+\frac{\gamma}{1+\gamma^{2}}\right)
$$

in which $\gamma=\left(2 v_{\mathrm{F}}\right) / \lambda$ and $\lambda \equiv \lambda_{\mathrm{TF}}=\left(4 v_{\mathrm{F}} / \pi\right)^{1 / 2}$, the so-called Thomas-Fermi screening parameter $[2,3,6]$. The Fermi velocity $\left(v_{\mathrm{F}}\right)$ and the density parameter $\left(r_{\mathrm{s}}\right)$ are defined as usual: $v_{\mathrm{F}}=\left(3 \pi^{2} n_{0}\right)^{1 / 3}$ and $r_{\mathrm{s}}=1.92 / v_{\mathrm{F}}$ at a given density $n_{0}$ of the $3 \mathrm{D}$ electron gas.

It is difficult to determine the accuracy of the mentioned approximations without going on, even with a fixed interaction between quasiparticles, to higher-order approximations. Furthermore, we note that a quasiparticle may, in a certain sense, be treated like a particle in the 'self-consistent' field of the other particles. But one should made allowance [7] not only for the effect of surrounding particles on the potential energy. It is the dynamical correlation of electrons which reduces the Coulomb repulsion between them. This aspect is not contained in a simple SCF approximation like the RPA. The added electron is a 'moving impurity' with velocity around $v_{\mathrm{F}}$. Clearly, the consistency problem, namely the interplay between the scattering and screening aspects, is not trivial.

Reliable data on the $a$-prefactor are given by experiments performed $[8,9]$ with cold ( $T=5 \mathrm{~K}$ ) scanning tunnelling microscopy (STM) on noble metal surfaces. The advantages of STM over different photoemission techniques are the capability

(i) to choose a spot bare of impurities (reduction of nonlifetime effects), and

(ii) to probe the electron dynamics (correlation) locally without depopulation and cascade problems.

Notice, in addition, a few important aspects. The probed portion of the band-related $k$-space is not exactly known and may depend on the tip geometry [10]. The other aspect is the usually neglected feedback effect of inelastic transitions. The injected electron and the decay process perturb the one-electron band structure back toward the free-electron-like band [11].

A quantitative prediction for the inelastic scattering rate has been subtracted (firstly in [8], using elastic tunnelling theory) from interference patterns generated on an $\mathrm{Ag}(111)$ surface and compared with the $\left(1 / \tau_{\mathrm{ee}}\right)=a \varepsilon^{2}$ expression down to about $\varepsilon=1 \mathrm{eV}$ excess energy. The subtraction is based on an analysis of measured amplitude damping in the oscillating local density of states by assuming a homogeneous medium in which local inelastic scatterings result in the (exponential) damping. It was explicitly stated that the data follow the $\left(E-E_{\mathrm{F}}\right)^{2}$-dependence, a 3D Fermi-liquid character. The 2D electron gas formed on noble-metal surfaces is surrounded by $3 \mathrm{D}$ electrons [12] which may essentially influence the screening and govern damping behaviours [8]. The predicted $a_{\exp }$-value for the Ag target is higher than the value that equation (1) would give with the usual $\lambda=\lambda_{\mathrm{TF}}$ for a $3 \mathrm{D}$ electron gas: $a_{\exp }(\mathrm{Ag})=1.58 a^{(1)}\left(r_{\mathrm{s}}=3\right)$. The enhancement $(1 \rightarrow 1.58)$ was attributed to the 
band structure and associated in-plane inelastic scattering effects [8]. The other experimental work [9], using the same subtraction tools, confirms the above $a_{\exp }(\mathrm{Ag})$ value down to $\epsilon=0.4 \mathrm{eV}$.

Any theoretical attempt, with the aim to explain, by one model assumption, the observed enhancement in $1 / \tau$ down to $\varepsilon=0.4 \mathrm{eV}$, is constrained with standard paradigms. The bandbased version of the RPA dielectric theory, namely the GW framework [13] for real targets, focuses on the role of specified (in $k$-space) single-particle states, usually the unperturbed ones in a Hartree-Fock-like picture [14]. Of course, it is expected that higher-order effects 'will certainly give numerical changes' [15] to this lowest-order treatment for an energy width $\Gamma(\sim \hbar / \tau)$.

The kinetic framework may allow more controllable modifications of the conditions stated at equation (1), but still uses the homogeneous liquid picture. As a conservative step in comparative studies (and motivated by the above-outlined experimental interpretation of measured amplitude damping), we shall use this framework and focus on the interplay between screening and scattering.

\section{Theory and results}

Since we are interested in decays close enough to the Fermi surface, all the colliding particles remain very close to this surface. The resulting decoupling (a characteristic feature of a degenerate system) of the angular and energy variables makes the calculation for $a\left(r_{\mathrm{s}}\right)$ easier. Denoting by $\theta$ the usual scattering angle, and by $\alpha$ the angle between the two incoming momenta in the centre-of-mass (c.m.) system, we can write [3, 4]

$$
a\left(r_{\mathrm{s}}\right)=\frac{1}{(2 \pi)^{4}}\left[\frac{w(\alpha, \theta)}{\cos (\alpha / 2)}\right]_{\mathrm{av}},
$$

in which $w(\alpha, \theta)$ is the transition probability for the collision process. By energy conservation, the scattering energy $\left(E_{\mathrm{c}}\right)$ in the c.m. system is determined via $\cos \alpha=1-\left(E_{\mathrm{c}} / E_{\mathrm{F}}\right)$.

The average (av) is defined [3, 4] as follows:

$$
\left[\frac{w(\alpha, \theta)}{\cos (\alpha / 2)}\right]_{\mathrm{av}}=\frac{1}{2 \pi} \int_{0}^{\pi} \mathrm{d} \theta \int_{0}^{\pi} \mathrm{d} \alpha \sin \alpha \frac{w(\alpha, \theta)}{\cos (\alpha / 2)} .
$$

In the knowledge of a screened (well-behaved) potential one can formulate $w(\alpha, \theta)$ in terms of a stationary scattering amplitude by using for it, e.g., a second-order Born approximation and (numerical) partial wave expansion.

In order to show the effect of the higher-order term clearly, we model the effective interaction by a Thomas-Fermi-type potential $V_{\mathrm{sc}}(r)=(1 / r) \exp (-\lambda r)$. For this potential form the required first- and second-order scattering amplitudes are well-known [16]. By using the real part of this amplitude one gets, up to the next-to-leading order, the following expression for the $w(\alpha, \theta)$ quantity:

$$
w(\alpha, \theta)=\frac{2 \pi(2 \pi / \mu)^{2}}{\left(q^{2}+\lambda^{2}\right)}\left[\frac{1}{\left(q^{2}+\lambda^{2}\right)}-2 \frac{2 \mu}{q f} \arctan \frac{q \lambda}{2 f}\right],
$$

in which $\mu=1 / 2$ is the reduced mass, $f^{2}=\lambda^{4}+4 \lambda^{2} k^{2}+k^{2} q^{2}$, with $k=v_{\mathrm{F}} \sin (\alpha / 2)$ and $q=2 k \sin (\theta / 2)$.

A restriction to the first term in the parentheses (first-order Born) gives equation (1) with the $\lambda$ screening parameter. The special sensitivity $\tau \sim \lambda^{3}$ is strong. A $25 \%$ increase in $\lambda$ gives a factor of two in $\tau$, showing the importance of a proper treatment of the screening parameter. The unmodified equation (1) could describe the $a_{\text {exp }}$-value with a certain $\lambda<\lambda_{\mathrm{TF}}$. On the 


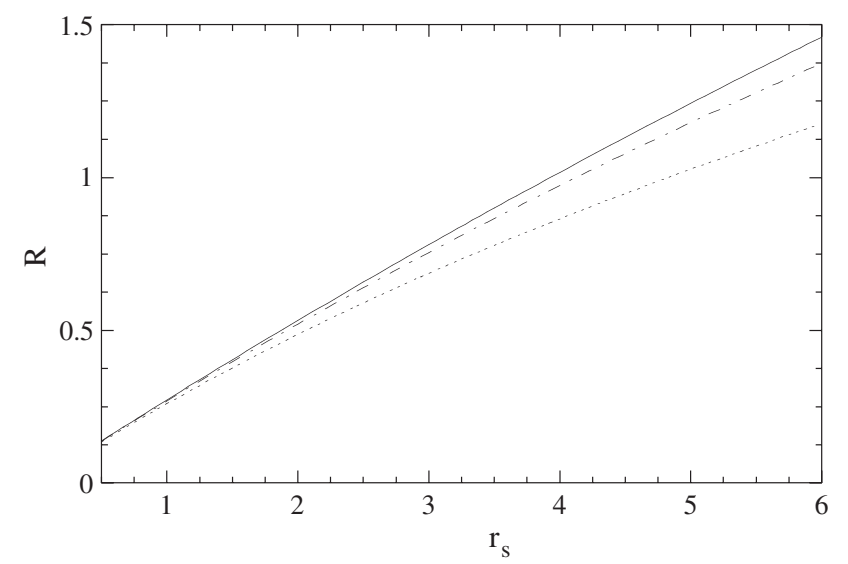

Figure 1. The ratio $R$ of the next-to-leading and the leading terms, obtained in a second-order description of the low-energy lifetime, as a function of the density parameter $r_{\mathrm{s}}$. The three options for the screening parameter, $\lambda_{\mathrm{TF}}, \lambda_{\mathrm{Y}}$, and $\lambda_{\mathrm{NP}}$ are referred by dotted, chain, and solid curves, respectively.

other hand, if the screening is relatively weak the applicability of a simple first-order Born approximation is in doubt [17]. By substituting the complete form of equation (4) into (3) one can perform numerical calculations and obtain the second-order Born result $\left[a^{(\mathrm{sB})}\left(r_{\mathrm{s}}\right)\right]$. Note that the $a^{(\mathrm{sB})}\left(r_{\mathrm{s}}\right)=a^{(1)}\left(r_{\mathrm{s}}\right)-a^{(2)}\left(r_{\mathrm{s}}\right)$ notation for the perturbative numerical results will be used below.

Now, we discuss the important question of choosing an input $\lambda\left(v_{\mathrm{F}}\right)$. The RPA-based long-wavelength (Thomas-Fermi limit) prescription gives the $\lambda_{\mathrm{TF}}=\left(4 v_{\mathrm{F}} / \pi\right)^{1 / 2}$ estimation. By considering the fact that an electron is not a static impurity, Young [18] arrived at the value $\lambda_{\mathrm{Y}}=(1 / 2)^{1 / 2} \lambda_{\mathrm{TF}}$. He employed a very sophisticated coordinate transformation on a Slater determinant in order to allow the proper consideration of dynamical correlation in the pair function of electrons. The variational method of Nozieres and Pines [19], by investigating the screened exchange and Coulomb correlation energies for metallic densities, results in an even more reduced average screening with $\lambda_{\mathrm{NP}} \cong 0.58 \lambda_{\mathrm{TF}}$. We shall use the $\lambda \in\left[\lambda_{\mathrm{NP}}, \lambda_{\mathrm{TF}}\right]$ range in our calculation on the observable quantity $a\left(r_{\mathrm{s}}\right)$.

The representative results are exhibited in three figures. In figure 1 we plot the ratio $(R)$, defined by $R=a^{(2)}\left(r_{\mathrm{s}}\right) / a^{(1)}\left(r_{\mathrm{s}}\right)$, as a function of the parameter $r_{\mathrm{s}}=(9 \pi / 4)^{1 / 3} / v_{\mathrm{F}}$. The three options, $\lambda_{\mathrm{TF}}, \lambda_{\mathrm{Y}}$, and $\lambda_{\mathrm{NP}}$ are referred by dotted, chain, and solid curves, respectively. Although the $a\left(r_{\mathrm{s}}\right)$ results are sensitive (see figures 2 and 3 ) to the particular value of $\lambda\left(r_{\mathrm{s}}\right)$, the ratio is quite insensitive to it. Motivated by figure 1 we approximate the second-order result with $a^{(\mathrm{sB})}\left(r_{\mathrm{s}}\right)=a^{(1)}\left(r_{\mathrm{s}}\right)\left[1-R\left(r_{\mathrm{s}}\right)\right]$. A simple relationship $R \cong(0.2-0.25) r_{\mathrm{s}}$ gives the possibility of accurate analytical estimations for different densities, at least within the limitation of a second-order approximation. Notice, parenthetically, that by a simple sign changing in the second term of equation (4), and thus of $R$, one could model the lifetime of an extended hole state at small $|\varepsilon|$.

The $R=1$ value, as the limit of physically meaningful results, appears at about $r_{\mathrm{s}} \approx 4-5$. The mathematical limitation, i.e., the problem of convergence [17] in an asymptotic series expansion like the Born series, puts down the validity limit of the quadratic description to about $r_{\mathrm{s}}=2$. Of course, the first-order result has an even more constrained limitation; it is valid at high densities for an acceptable screening: $\lambda \leqslant \lambda_{\mathrm{TF}}$. 


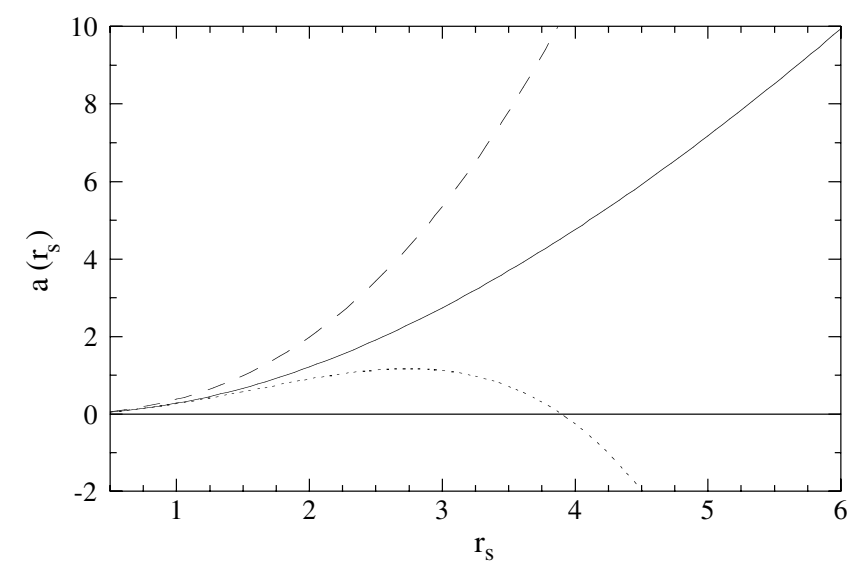

Figure 2. The calculated $a\left(r_{\mathrm{s}}\right)$ functions in different scattering approximations, with $\lambda_{\mathrm{NP}}\left(r_{\mathrm{s}}\right)$ screening parameter. Solid curve: phase-shift-based determination of the scattering amplitude. Dashed and dotted curves: first- $\left[a^{(1)}\left(r_{\mathrm{s}}\right)\right]$ and second-order $\left[a^{(\mathrm{sB})}\left(r_{\mathrm{s}}\right)\right]$ Born approximations.

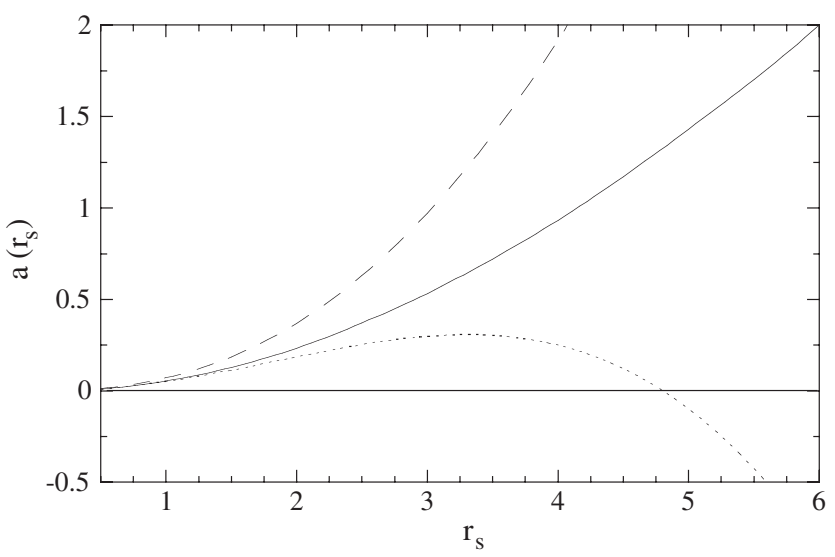

Figure 3. The same as in figure 2, with $\lambda_{\mathrm{TF}}\left(r_{\mathrm{s}}\right)$ screening.

The above statements are illustrated in detail in figures 2 and 3. They show, for the important range of $r_{\mathrm{s}}$, the results obtained for $a\left(r_{\mathrm{s}}\right)$ in different scattering approximations by using $\lambda_{\mathrm{NP}}\left(r_{\mathrm{s}}\right)$ and $\lambda_{\mathrm{TF}}\left(r_{\mathrm{s}}\right)$ screening parameters, respectively, as limiting values for $\lambda$. The solid curves are based on numerical solutions for phase shifts in order to describe [5] the scattering amplitudes in the partial-wave representation:

$$
f(\theta)=\frac{1}{\sqrt{E_{\mathrm{c}}}} \sum_{l=0}^{\infty}(2 l+1) \mathrm{e}^{\mathrm{i} \delta_{l}} \sin \delta_{l} P_{l}(\cos \theta),
$$

in which $\delta_{l}\left(E_{\mathrm{c}}\right)$ are the numerically determined phase shifts. Thus the transition probability to be introduced in equation (3) is [20] $w(\alpha, \theta)=2 \pi(2 \pi / \mu)^{2}|f(\theta)|^{2}$. The dashed and dotted curves in these figures refer, respectively, to the first- $\left[a^{(1)}\left(r_{\mathrm{s}}\right)\right]$ and second-order $\left[a^{(\mathrm{sB})}\left(r_{\mathrm{s}}\right)\right]$ Born expansions for the scattering amplitude. The second-order treatment gives improvements, but at lower densities a nonperturbative estimation is needed for the relaxation time. 
Remarkably, a simple expression of practical interest,

$$
a\left(r_{\mathrm{s}}\right)=\frac{a^{(1)}\left(\lambda, r_{\mathrm{s}}\right)}{1+0.25 r_{\mathrm{s}}}
$$

gives a very accurate fit to both solid curves of figures 2 and 3 . This equation allows a transparent interpretation: the nominator, $a^{(1)}\left(\lambda, r_{\mathrm{s}}\right)$ of equation (1), encodes the special screening sensitivity ( $a \sim \lambda^{-3}$; see the figures) and the denominator corrects its perturbative nature. It turns out that the physically best-motivated $\lambda_{\mathrm{Y}}=1.1 / \sqrt{r_{\mathrm{s}}}$ prescription results in $a_{\text {theor }}\left(r_{\mathrm{s}}=3\right) \cong a_{\exp }(\mathrm{Ag})$, using the phase-shift-based expression of equation (6). This theoretically motivated reduced screening parameter is in complete agreement with a phenomenologically introduced one [21]. The authors of [21] also point out the importance of the reduction in modelling interaction potentials in silver and gold nanoparticles.

We should note, for completeness, that for $\varepsilon \in[0.1,0.4] \mathrm{eV}$ the experimental data [9] herald a further lowering in the subtracted lifetime $(\tau)$. This is, in fact, the energy range in which the unperturbed (ground state) one-particle surface state exists. Supposing that the relaxation length $L_{\phi}(\sim \tau)$ fitted in $[8,9]$ and, therefore, the subtracted exponential amplitude damping is still not influenced by the step position, one may attribute the deviation to an $\varepsilon$-independent, i.e., phonon-assisted attenuation $1 / \tau_{\mathrm{ep}}$. Under the experimental conditions, $T \ll \omega_{\mathrm{D}} \ll \varepsilon$ (where the Debye frequency is about $\omega_{\mathrm{D}}=20 \mathrm{meV}$ ), this channel gives [22] a constant contribution which can be added to the electron-electron part at low $\varepsilon$-values, using the $(1 / \tau)=\left(1 / \tau_{\mathrm{ee}}\right)+\left(1 / \tau_{\mathrm{ep}}\right)$ expression. We state that with a small constant value of about $\left(1 / \tau_{\mathrm{ep}}\right) \simeq 2.5 \mathrm{meV}$, one could describe the data [8,9] down to $\varepsilon=0.1 \mathrm{eV}$. This small value seems to be consistent with an electron-gas-like behaviour. It is about $2 / 3$ of the previously applied [9] value for $1 / \tau_{\text {ep }}$, obtained by using a linearly screened pseudopotential [23]. A quadratic effect in the effective valence screening might be a possible source in coupling reduction (see also [21]).

\section{Conclusions}

The present theoretical attempt is based on the Fermi-liquid model. Therefore, making predictions for experiments or drawing quantitative conclusions about the interactions in a given metal proves possible only in exceptional cases, i.e., for quasi-homogeneous systems. As this $i s$ the experimental situation, we consider the agreement for $\tau_{\mathrm{ee}}$ as an important step in comparative studies on a fundamental one-particle characteristic of many-body origin. The important ingredients of our consistent description are the proper screening parameter and the nonperturbative treatment of electron-electron scattering.

In addition, we remark that in the density-fluctuation-based many-body approximation, the GW [13] method, attempts to go to higher order in a perturbation expansion are rare; for a recent detailed discussion see [24]. The coupled Hedin's equations are integro-differential equations and, therefore, require a nontrivial mathematical analysis in formal expansions [25]. The kinetic framework, applied in this work, indicates that the interplay between screening and scattering (see equation (6)) is a nontrivial question for dynamically correlated particles in their environment. Any theoretical attempt should consider this consistency problem.

\section{Acknowledgments}

MA and JIJ acknowledge the supports from UPV/EHU, Eusko Jaurlaritza, Ministerio de Educacion y Cultura (Grant No. BFM2001-0076). MA also acknowledges financial support by the Gipuzkoako Foru Aldundia. The work of IN has been supported by the Hungarian OTKA 
(Grant Nos T034363 and T038162). He thanks A Zawadowski for useful conversations on the medium-particle aspects of the decay. We are grateful for discussions with E V Chulkov, P M Echenique, A Eiguren, and V M Silkin.

\section{References}

[1] Luttinger J M 1961 Phys. Rev. 121942

[2] Quinn J J and Ferrell R A 1958 Phys. Rev. 112812

[3] Pines D and Nozieres P 1982 The Theory of Quantum Liquids (New York: Benjamin) sections 1.8 and 5.5

[4] Morel P and Nozieres P 1962 Phys. Rev. 1261909 Giovanardi N 2001 Eur. J. Phys. 22185

[5] Nagy I, Juaristi J I and Echenique P M 2000 Phys. Rev. B 63035102

[6] Ritchie R H 1959 Phys. Rev. 114644

[7] Lifshitz E M and Pitaevskii L P 1995 Statistical Physics vol 2 (Oxford: Butterworth-Heinemann) chapter 1 Fulde P 1995 Electron Correlation in Molecules and Solids (Berlin: Springer) section 2.8

[8] Bürgi L, Jeandupeux O, Brune H and Kern K 1999 Phys. Rev. Lett. 824516 Bürgi L 1999 PhD Thesis Swiss Federal Institute of Technology, Lausanne

[9] Vitali L, Wahl P, Schneider M A, Kern K, Silkin V M, Chulkov E V and Echenique P M 2003 Surf. Sci. 523 L47

[10] Bauer A, Mühlig A, Wegner D and Kaindl G 2002 Phys. Rev. B 65075421

[11] Goldmann G, Altmann W and Dose V 1991 Solid State Commun. 79511

[12] Bürgi L, Brune H and Kern K 2002 Phys. Rev. Lett. 89176801

[13] Hubbard J 1957 Proc. R. Soc. A 240539 Hedin L 1965 Phys. Rev. A 139796

[14] Echenique P M, Pitarke J M, Chulkov E V and Rubio A 2000 Chem. Phys. 2521 and references therein

[15] Hedin L and Lundqvist S 1969 Solid State Physics vol 23, ed H Ehrenreich, F Seitz and D Turnbull (New York: Academic)

[16] Newton R 1982 Scattering Theory of Waves and Particles (New York: Springer) equation (10.135)

[17] Nozieres P 1997 Theory of Interacting Fermi Systems (Reading, MA: Addison-Wesley) chapter 5

[18] Young W H 1963 Phys. Rev. 1292019

[19] Nozieres P and Pines D 1958 Phys. Rev. 111442

[20] Schiff L I 1968 Quantum Mechanics (New York: McGraw-Hill) equations (37.3) and (37.21)

[21] Arbouet A et al 2003 Phys. Rev. Lett. 90177401

[22] Abrikosov A A, Gorkov L P and Dzyaloshinski I E 1975 Methods of Quantum Field Theory in Statistical Physics (New York: Dover) equation (21.42)

[23] Hellsing B, Eiguren A and Chulkov E V 2002 J. Phys.: Condens. Matter 145959

[24] Aulbur W G, Jonsson L and Wilkins L W 2000 Solid State Physics ed H Ehrenreich and F Spaepen (San Diego, CA: Academic) vol 54

[25] Farid B 1999 Electron Correlation in the Solid State ed N H March (London: Imperial College Press) 